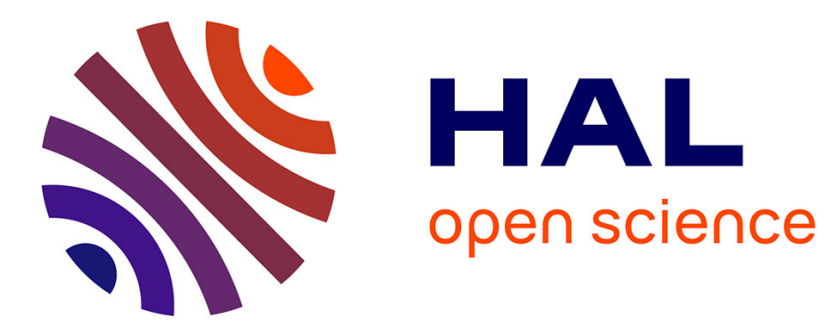

\title{
Superpixel-based Zernike Moments for Palm-print Recognition
}

Youssef Chahir, Amina Serir, Bilal Attallah, Abdelwahhab Boudjelal

\section{To cite this version:}

Youssef Chahir, Amina Serir, Bilal Attallah, Abdelwahhab Boudjelal. Superpixel-based Zernike Moments for Palm-print Recognition. International journal of electronic security and digital forensics, 2019, Vol.11 (No.4), pp.420 - 433. 10.1504/IJESDF.2019.102561 . hal-01877629

\section{HAL Id: hal-01877629 \\ https://hal.science/hal-01877629}

Submitted on 27 Sep 2018

HAL is a multi-disciplinary open access archive for the deposit and dissemination of scientific research documents, whether they are published or not. The documents may come from teaching and research institutions in France or abroad, or from public or private research centers.
L'archive ouverte pluridisciplinaire HAL, est destinée au dépôt et à la diffusion de documents scientifiques de niveau recherche, publiés ou non, émanant des établissements d'enseignement et de recherche français ou étrangers, des laboratoires publics ou privés. 


\title{
Superpixel-based Zernike Moments for Palm-print Recognition
}

\author{
Bilal Attallah, ${ }^{\mathrm{a}, \mathrm{b}, \star}$ Amina Serir, ${ }^{\mathrm{a}}$ Youssef Chahir, ${ }^{\mathrm{b}}$ and Abdelwahhab Boudjelal ${ }^{\mathrm{b}}$ \\ aUSTHB, LTIR, Electronic Department, Algeria \\ bNormandy University, UNICAEN, ENSICAEN, CNRS, GREYC, Caen, France
}

\begin{abstract}
In the contemporary period, significant attention has been focused on the prospects of innovative personal recognition methods based on palm print biometrics. However, diminished local consistency and interference from noise are only some of the obstacles that hinder the most common methods of palm-print imaging such as the grey texture and other low-level of the palm. Nevertheless, the development of the process and tackling of the obstacles faced have a potential solution in the form of high-level characteristic imaging for palm-print identification. In this study, Zernike Moments are used for acquiring superpixel features that are spiral scanned images, which is an innovative recognition method. By using the extreme learning machine, the inter- and intra-similarities of the palm-print feature maps are determined. Our experiments yield good results with an accuracy rate of 97.52 and an equal error rate of $1.47 \%$ on the palm-print PolyU database.
\end{abstract}

Keywords: palm-print recognition, image segmentation, feature extraction, ELM, image matching.

\section{Introduction}

As a means of enhancing palm-print recognition precision, the development of strong algorithms has been the focus of biometric analysts over the last twenty years. Varying degrees of user tolerability, effectiveness, and precision have been observed with the use of palm-print, hand, face, voice, iris, and fingerprint biometric modalities. Owing to the recently reduced expenditure required for the sensors and high user tolerability of palm-print identification methods, the current biometric modalities are now being increasingly considered for supplementation with this original and promising biometric mechanism. Fingerprinting is often found to be more disconcerting for individuals than palm printing. Furthermore, individuals have been perceived to be concerned when an identification device requires that the eyeball of a user be scanned and iris calculations be performed. An additional advantage of palm printing is that a myriad of significant textures, ridges, creases, and lines that make for distinct characteristics exist on a palm, and it also presents a large surface for identifying such characteristics in contrast with various other biometric modalities.

The prospects for various biometric systems that make use of palm prints have been explored contemporarily; appearance-based and structural-based systems are the two primary types of biometric systems that have been identified. In the case of the structural-based systems, an array of edge identification mechanisms can be adopted to acquire a palm-print scan's distinctive lines and ridges (Wua et al. 2004;Han et al. 2003). Owing to the typical characteristics of a palm of having ridges and wrinkles that intersect and the difficulty in producing an image of such features, the precision of the characteristic identification method is a highly significant aspect of performance according to existing studies. The efficiency of the use of palm-print lines for identifying a user, on having extracted its features by using an amended Radon transform, was confirmed in the contemporary research performed 
by (Huan et al. 2008). However, in order to resolve the issue of the deficient abstraction of the palm-print line features in the region of interest (ROI), an amended finite Radon transform with a larger training array for acquiring the orientation code was proposed by ( Jai et al. 2008).

In the case of appearance-based systems, a high-dimensional issue is regularly produced owing to the two-dimensional intensity variation features being treated and evaluated as a sole dataset in relation to the palm print. Therefore, the identification procedure should be improved in terms of precision through a crucial stage that involves transforming the high-dimensional palm-print information into a lowdimensional image. The primary statistical consistencies of the image can be determined and quantified as a means of conducting palm-print identification using such methods; both unsupervised and supervised classifications can be distinguished in this manner. The local binary pattern (LBP) (Hanmandlu et al. 2010) and principal component analysis (PCA) (Lu et al. 2003; Zhao et al. 2007; Ribaric et al. 2005) are two approaches that can be adopted to acquire the necessary data without the use of data label indexing for the unsupervised classifications. In the case of the supervised classifications, linear discriminant analysis and other common statistical means can be adopted for high-dimensionality reduction of the training phase's characteristic vectors while cataloguing on the basis of the predetermined class labels (Wue et al. 2003;Jing et al. 2004). Maintaining the information's local structure through low-dimensional imaging has been contemporarily attempted using the LBP approach. However, the importance of the discriminant data, which is crucial to the identification phase, is reduced with the use of this method.

Individual pixel processing of representations within the spatial dimension has largely characterized appearance-orientated systems. However, if flaws exist, several disadvantages are posed by such methods. The enhancement of the discernment capabilities is possible by altering a basic characteristic selection data in the frequency domain through the use of common transform methods (Laadjel et al. 2008; Naani et al.2008). The defining characteristic data was acquired by using an enhanced form of LDA (Linear discriminant analysis) and choosing the DCT (discrete cosine transform) frequency bands using a two-dimensional separability judgment algorithm with the use of a 3040 palm-print scan database showing positive outcomes in Xiao et al.'s research (Jing et al.2004). In order to enhance the identification precision and reduce the expenditure on computation methods, an LDA was used for characteristic discernment across five representation levels of the palm print in Xiangqian et al.'s study using a Gaussian pyramid to produce the five levels and reduce the resolution (Wue et al. 2003). Furthermore, the high-dimensionality reduction of the characteristic space was also undertaken by implementing 2DPCA+PCA in Xin et al.'s study after acquiring the local characteristics by using Gabor filters, which decomposed the palm-print representations into various orientations and scales. However, the implementation of the Gabor filter bank causes the problem of exploding dimensionality, which is the major disadvantage of this approach (Pan et al. 2008). Furthermore, by incorporating independent component analysis (FASTICA) with radial basis probabilistic neural networks (RBPNN), an innovative palm-print identification method was devised by Li-Shanga et al. However, the larger the database, the greater the decline observed in terms of magnitude and severity in identification precision, while the computational requirements are significant (Shang et al. 2006). Individual identification on the basis of degraded palm-print images has not been an issue in any of the researches outlined above, where algorithms were analyzed using clean palm-print databases. Overall, the identification success is most likely to be undermined by the degradation of the palm-print images as a result of distortion or noise interference. 
An array of obstacles is faced in LLF-based approaches despite various studies providing satisfactory experimental outcomes on this subject (Wua et al. 2004;Jai W et al.2008). For example, the local consistency features of palm prints cannot be defined using low-level characteristics. Furthermore, representation discrepancies typically affect the corresponding algorithms, such as the Hamming distance algorithm, while low-level characteristic palm-print systems are vulnerable to pixel noise (Shang et al.2006).

Therefore, implementing Zernik moments in relation to the superpixel region is the novel approach presented in this research in order to overcome the aforementioned obstacles. The palm-print PolyU database showed a mere $1.47 \%$ equal error rate (EER), and thus, some excellent results were obtained in this research.

The ability of the aforementioned approach of acquiring all the palm data associated with the palm surface in order to produce a superpixel-based characteristic acquisition algorithm is the primary contribution of this study. Section 2 provides an in-depth explanation of the method adopted in this research. The experimental outcomes are outlined and evaluated in section 3, while section 4 presents the conclusions.

\section{Methodology}

Figure 1 introduces the SPC (superpixel scheme)-based original palm-print identification system. Having provided an overall summary of the devised system in figure 1, the distinct stages of the system can be identified. First, pre-processing of the representation occurs, which includes image normalization, ROI extraction, and image alignment. Second, in order to produce the superpixels, the simple linear iterative clustering (SLIC) technique (Achanta et al. 2012) was adopted during the superpixel oversegmentation stage. Third, superpixel feature (SPF) extraction is performed using Zernike moments in order to acquire the superpixel characteristics (Kal et al.2 013). Fourth, feature normalization is performed (Aksoy et al. 2000). The final stage comprises the matching score calculation with template palm-print representations, and the input's standardized characteristics are used to calculate the matching score by using an extreme learning machine (ELM) (Huang et al. 2006).

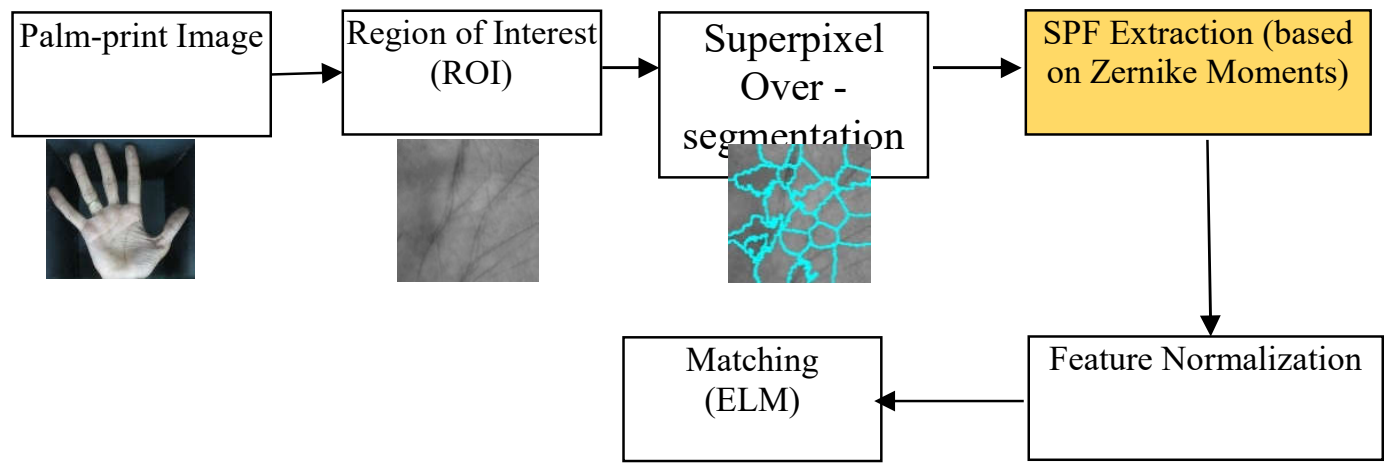

Fig. 1 Palm-print identification system summary 


\subsection{Extraction of the Region of Interest}

As a means of straightening the palm-print representation, the key points were identified based on the technique explained for key point identification in (Zhang et al. 2003). The acquisition of a $128 \times 128$ size ROI was possible after the palm-print representation had been aligned. Figure 2 presents the extracted ROI and a palm-print example.

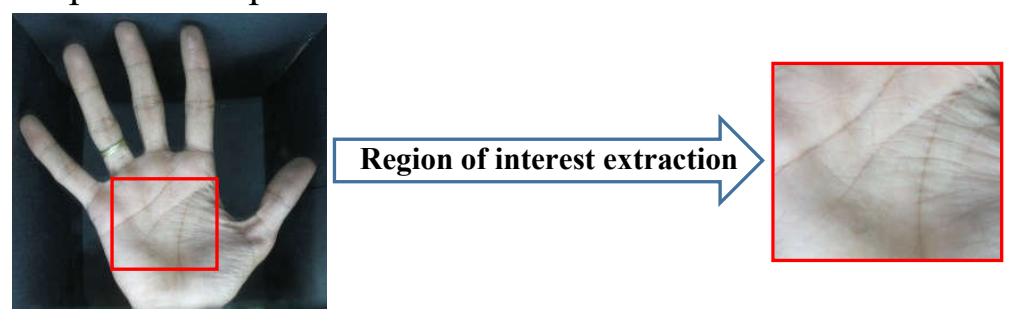

Fig. 2 Extraction of the ROI

\subsection{Over-segmentation of Superpixels}

Superpixels are typically acquired through superpixel over-segmentation. The production of superpixels via finger-vein representation over-segmentation was undertaken using the SLIC technique (Achanta et al. 2012) . The location data and local intensity are merged to produce small areas of the representation that were identified using SLIC. The advantages of using this technique are the reduced computational requirements and the requisite superpixel amount being produced based on a single particular parameter. There are two crucial points of departure from the k-means of the superpixel generation in the case of SLIC. Firstly, the superpixel size is equal to the constraints of the search area, thus considerably diminishing the total distance measurements of the optimization. Furthermore, the complexity is independent of the number of superpixels while also having linearity with the amount of pixels. Secondly, the regulation of superpixel density and size is possible, in addition to an amalgamation of the spatial and color closeness, by using the weighted distance measure.

If the requisite amount of superpixels is represented by $k$, while the amount of pixels in the representation area is represented by $n$, then a superpixel-size equivalent area will be restricted for search in the SLIC. Thus, $s=\sqrt{n / k}$, and $s \times s$ is the rough size of the superpixel area, while the grid interval is represented by $S$. Ultimately, as shown in Eq. (1), the weighted distance measure $D$ is used to determine the amalgamation of the image plane and color space for the pixel grouping via SLIC. This produces superpixels that are dense and almost standardized.

$$
\begin{gathered}
D=\sqrt{\left(\frac{d_{c}}{N_{c}}\right)^{2}+\left(\frac{d_{s}}{N_{s}}\right)^{2}} \\
d_{c}=\sqrt{\left(l_{i}-l_{j}\right)^{2}} \\
d_{s}=\sqrt{\left(x_{i}-x_{j}\right)^{2}+\left(y_{i}-y_{j}\right)^{2}}
\end{gathered}
$$


With the normalized factors being $N_{C}$ and $N_{S}$, the space proximity is represented by $d_{s}$, the color proximity is represented by $d_{c}$, the cluster centers are represented by $\left[l_{k} x_{k} y_{k}\right]$, the pixel location is represented by $\left[\begin{array}{ll}x & y\end{array}\right]$, and colored regions of the greyscale representations are represented by $l^{T}$.

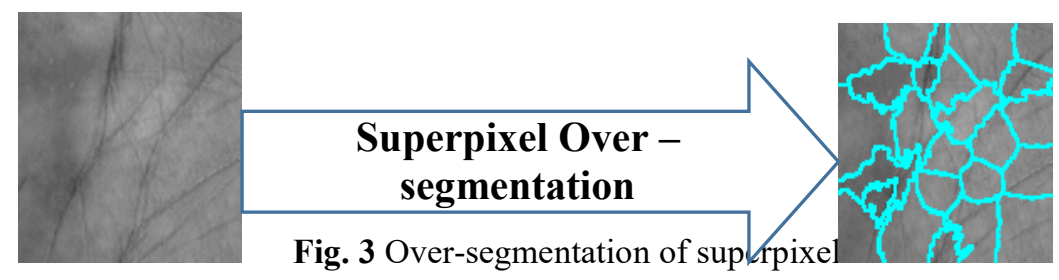

\subsection{Characteristics Extraction using Zernike Moments}

The irregular partitions of the representation are indicated by the region adjacency graph (RAG), which has $\mathrm{E}$ edges and $\mathrm{V}$ vertices and is an undirected graph $\mathrm{G}$. The feature vector $\mathrm{F}(\mathrm{v})$ is present for every participation while also possessing a vertex (v).

A precise characteristic descriptor was produced and an array of invariants devised by means of twodimensional Zernike moments (2DZMs). Inside unit disk D, the polar coordinates $(\rho, \theta)$ are adopted to characterize the 2DZMs of the $p$ th order possessing repetition $q$.

$$
D=\{(p, q)|0 \leq p \geq \omega,| q|\leq p,| p-q \mid \text { is even }\}
$$

and

$$
Z_{p q}=\frac{p+1}{\pi} \sum_{x} \sum_{y} V_{p q}^{*}(x, y) f(x, y)
$$

The definition of the Zernike polynomials can be represented as follows:

$$
V_{p q}^{*}(\rho, \theta)=R_{p q}(\rho) e^{i q \theta}
$$

The complex conjugate is denoted by the asterisk.

The vector's length from origin to pixel $(x, y)$ forms the radius $\rho=\sqrt{x^{2}+y^{2}}$, while the angle formed by the $\mathrm{x}$-axis and the vector is represented by $\theta=\arctan (y / x)$. The Zernike radial polynomial's path order is $R_{p q}$, which can be determined as follows:

$$
R_{p q}(\rho)=\sum_{k=|q||| p-q \mid i_{\text {seven }}}^{p} \frac{(-1)^{\frac{p-k}{2}} \frac{p+k}{2} !}{\frac{p-k}{2} ! \frac{k-q}{2} ! \frac{k+q}{2} !} \rho^{k}
$$


In order to describe the representation, it is essential that the maximum order of the 2DZMs is selected. When presented in the form of geometric movements, the second- and third-order Zernike invariants were found to be equal to the $\mathrm{Hu}$ invariants (Kale et al. 2013).

\subsection{The Region Adjacency Graph's spiral order traversal}

In this section, the results obtained when the superpixel area is subjected to spiral scanning are presented

As a means of forming a spiral path of successive vertices, the central partition was selected as the initial point for spiral scanning (Trémeau and colantoni. 2000) (see figure 4).

The idea of the algorithm is simple: the regions in the image are first numbered in spiral order from the center of the image. Each node (region) of the graph (i.e., RAG) that is represented by its barycenter corresponds to a number (in spiral order) in the image. This list is then sorted in alphanumeric order starting with the index 1 . Thus, all the nodes of the graph are labeled in ascending order in the spiral direction.

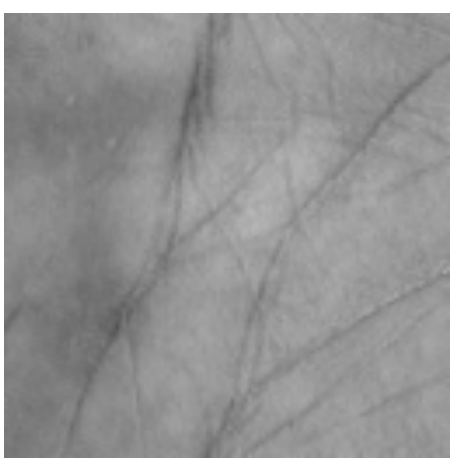

(a)

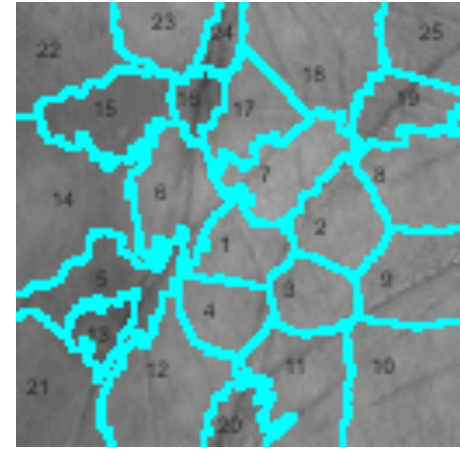

(b)

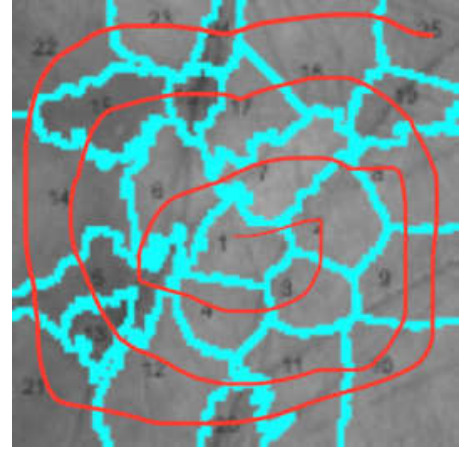

(c)

Fig. 4 (a). Palm-print representation, (b). Superpixel region, and (c). Spiral order of super-pixel region

\subsection{Feature Vector Normalization}

Feature vectors are irreconcilable owing to the distribution and range variability across them. However, feature vector normalization can be implemented using a normalization system (Aksoy and haralick. 2000) such as the median, z-score, or min-max system in order to remedy the aforementioned issue. The feature vectors across the range $[0,1]$ were normalized using the min-max system. On applying the min-max normalization, the normalized feature vector $X^{\prime}$ can be denoted as shown below when the feature vector is $X=\left[x_{1}, x_{2}, x_{3}, \ldots . ., x_{n}\right]$.

$$
X^{\prime}=\frac{x_{i}-\operatorname{Min}(X)}{\operatorname{Max}(X)-\operatorname{Min}(X)}
$$

\subsection{Extreme Learning Machine}

Single-hidden-layer feedforward neural networks have often been learnt using ELM (Huang et al. 2006). The iterative tuning of obscured nodes is not necessary following the random initialization through ELM. Thus, learning is required to occur just for the input weight parameters. If 
$\left(x_{j}, y_{j}\right), j=[1, \ldots, q]$ indicates $A$ as the training sample, with $x_{j} \in R^{M}$ and $y_{j} \in R^{M}$, then the formula below can be provided for the ELM's output function with L obscured neurons:

$$
f_{l}(x)=\sum_{i=1}^{L} g_{i} w_{i}(x)=\Omega(x) G
$$

The $m>1$ output nodes are associated with $L$ hidden nodes with the output weight vector $G=\left[g_{1}, \ldots, g_{L}\right]$. The nonlinear activation function is represented by $\Omega(x)=\left[w_{1}(x), \ldots, w_{L}(x)\right][14]$. The following formula is a variation of the explicit form of the system $\Omega_{i}(x)$ :

$$
\Omega_{i}(x)=\beta\left(\tau_{i} \cdot x+\theta_{i}\right), \tau_{i} \in R^{d}, \theta_{i} \in R
$$

The obscured layer parameters $(\tau, \theta)$ have the activation function $\beta($.$) . The following least square$ norm was used in order to resolve the output weight $\Omega$ and minimize the training data error during the second phase of the ELM learning:

$$
\min \|\Omega-H\|^{2}, G \in R^{N^{*} M}
$$

The hidden-neurons layer system is identified by $\Omega$ :

$$
\Omega=\left(\begin{array}{ccc}
\beta\left(\tau_{1} \cdot x_{1}+\theta_{1}\right) & \ldots & \beta\left(\tau_{L} \cdot x_{1}+\theta_{L}\right) \\
\vdots & \ddots & \vdots \\
\beta\left(\tau_{1} \cdot x_{N}+\theta_{1}\right) & \cdots & \beta\left(\tau_{L} \cdot x_{N}+\theta_{L}\right)
\end{array}\right)
$$

While the training data matrix $\mathrm{H}$ can be presented as follows:

$$
H=\left[\begin{array}{l}
h_{1}^{T} \\
\vdots \\
h_{N}^{T}
\end{array}\right]
$$

The training set (i.e., $L\langle Q$ ) values are greater than the amount of hidden neurons $\mathrm{L}$, which is assumed to be the case in reality, in order for the training error in Eq. (11) to be reduced in the optimal solution. (Huang et al. 2006) presents the optimal solution for Eq. (11), in the form of the Moor-Penrose generalized inverse matrix, where the inverse of $\Omega$ is $\Omega^{*}$.

\section{Experimental results}

The PPDB (PolyU Palm-print database) is an open domain, vast-scale database that was used as the basis of the comprehensive experiments undertaken in order to confirm the system's validity (). Alongside the statistical legitimacy, the recognition rate and EER are the two terms used to present the outcomes. 


\subsection{PolyU palm-print database}

For the PolyU palm-print database, two distinct sessions were held to obtain 20 palm-print images from 352 people. The test samples were those obtained during the second stage and comprised 10 images, while the control samples were those that were obtained during the initial session with 10 images per individual ( Zhang et al. 2010).

\subsection{Critical Evaluation in Relation to Existing Methods}

In this study, the SPF extraction is conducted by using Zernike moments from the last feature vector. To obtain the best results, superpixels of various sizes were selected. The degree of accuracy versus the superpixel number is shown in figure 5.

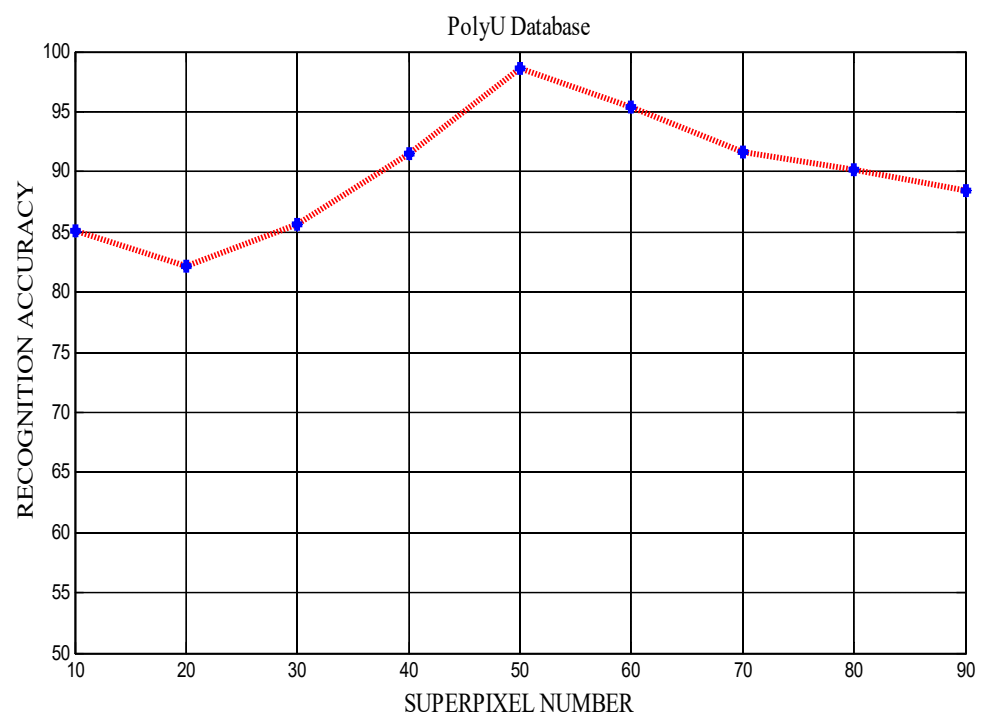

Fig. 5 Average accuracy for various superpixel numbers

Table 1 lists the PolyU PPDB database results obtained on applying the superpixel descriptor technique, and the effectiveness of this technique can thus be determined. With an EER of 2.76\%, this experimental technique was found to be superior to other feature extraction mechanism (Kong et al.2004; Kumar an Shen.2004; Sun et al.2005; Kong et al. 2006).

Table.1. PolyU palm-print databank and the proposed technique for measuring effectiveness

\begin{tabular}{|c|c|c|c|c|c|c|c|c|}
\hline EERs & $\begin{array}{c}\text { Competitive } \\
\text { code }\end{array}$ & $\begin{array}{c}\text { Palm } \\
\text { code }\end{array}$ & $\begin{array}{c}\text { Fusion } \\
\text { code }\end{array}$ & $\begin{array}{c}\text { Ordinal } \\
\text { code }\end{array}$ & RLOC & BOCV & $\begin{array}{c}\text { Half - } \\
\text { orientation }\end{array}$ & Proposed \\
\hline PolyU & 0.0261 & 0.0931 & 0.0899 & 0.0272 & 0.0360 & 0.0469 & 0.0204 & 0.0147 \\
\hline
\end{tabular}

The known class labels of a restricted number of palm-print images are used as training samples for the palm-print identification, while the remainder were unknown and were used as probe samples. The aim of the process is to predict the probe-image class labels by comparing images with the training samples using a one-against-many comparison. The EER (equal error rate) in the identification process is used to estimate the performance of the palm-print identification.

The probe samples are classified such that they are associated with the class of training samples to which they are most similar. In this study, the training samples were considered each palm's first $\mathrm{N}_{\text {train }} \in$ 
$\{2,4,6\}$ palm-print image(s). The probe palm prints were considered to be the remainder of the palmprint images. $\mathrm{N}_{\text {train }} \times \mathrm{N}_{\text {palm }}$ matching scores were generated in a database based on the probe sample.

The proposed method was then compared with several state-of-the-art orientation-based techniques, including the ordinal code (Sun et al.2005), fusion code (Kong et al. 2006), RLOC (Guo et al. 2009), palmCode (Kumar an Shen.2004), BOCV (Guo et al. 2009), and half-orientation methods (Fei and Zhang. 2016) (see figure 6).

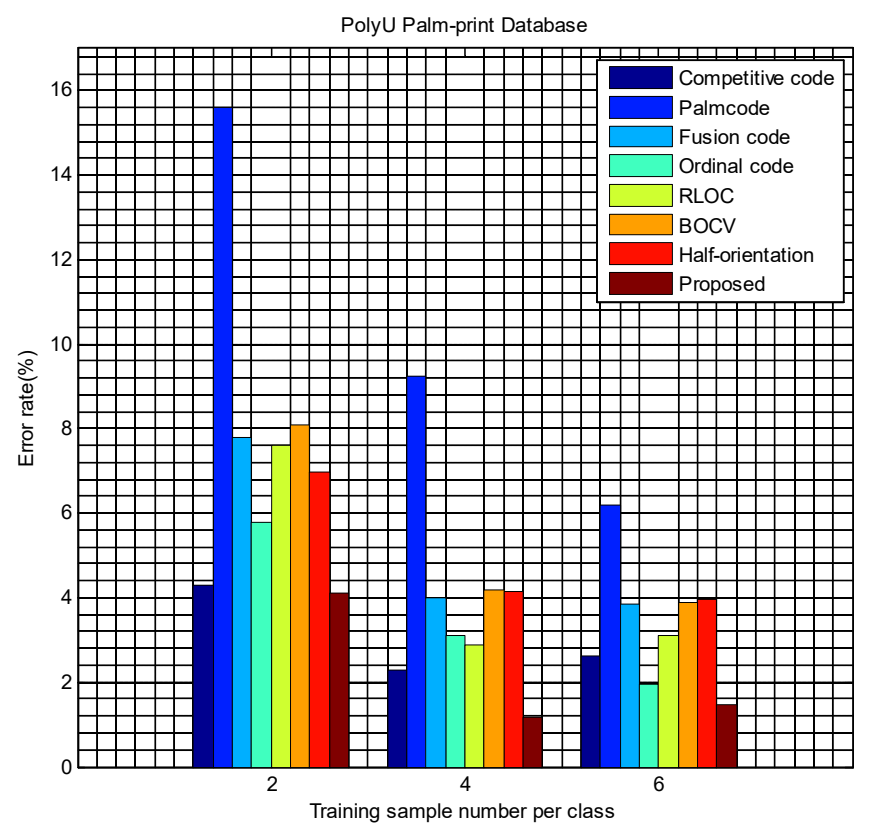

Fig. 6 EERs for PolyU palm-print databases

Several training palm-print images (between two and six for each palm-print database) were used to evaluate the proposed method's performance. The test dataset comprised the remainder of the palm-print images. Therefore, the identification experiments were performed six times with Ntrain $=1$ to 6 .

Figure 6 shows the results. It is evident that the proposed method is superior to other orientation-based methods when it is applied to the PolyU databases; it achieved lower error rates in both instances. The competitive code method extracted robust orientation features from the images with a high level of identification accuracy in specific instances. However, for the majority of the conditions, the proposed method was found to be superior to the competitive method.

In order to gauge the accuracy of the technique, we used various numbers of superpixels, and the results are shown in figure 7 . The EER results reveal that the most efficient number of superpixels was 50 for the PolyU databases. 


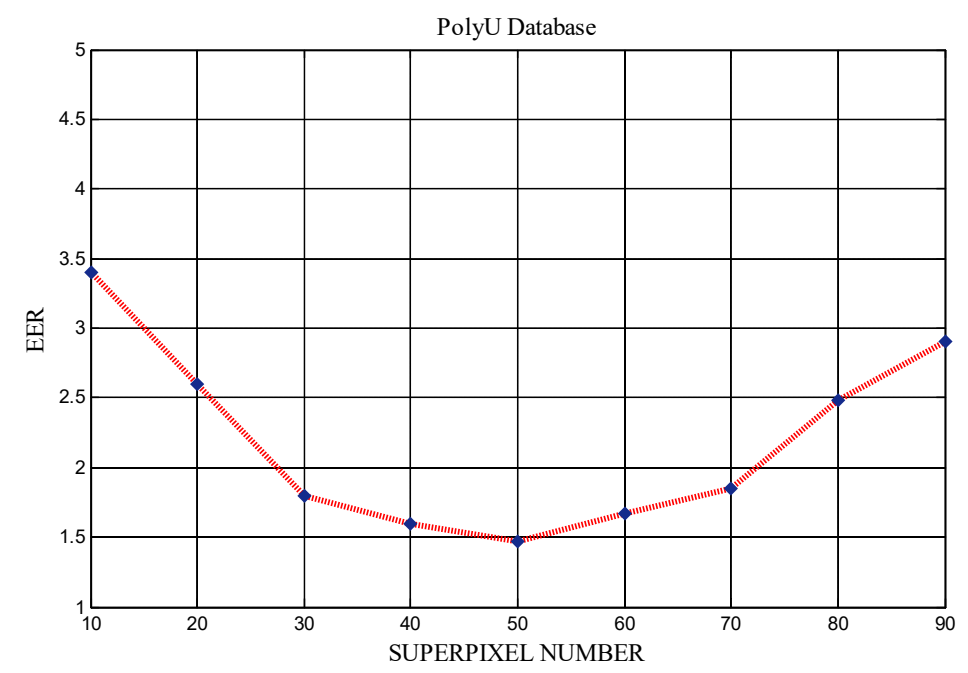

Fig. 7 EERs for various superpixel numbers

\subsection{Speed Effectiveness}

The proposed technique was implemented in MATLAB version 10.3 using a PC with a dual-core Intel i5-6600 3.3-GHz processor and 8-GB RAM. The operating system of the PC was Windows 10.0. The method was compared to previous state-of-the-art methods based on feature extraction in terms of computational cost in order to evaluate the complexity of its computation. The average time required to perform the feature extraction and matching over 100 runs was calculated to assess the computational complexity. The results thus obtained are shown in Table 2. The proposed method was found to consume a greater amount of time during feature extraction than the competitive code, BOCV, and half-orientation methods. However, the proposed method's matching speed was slightly higher as a simple matching scheme was used.

The proposed method required $26.228 \mathrm{~s}$ to perform the operation. This is acceptable for practical applications, although it is slightly greater than the time consumed by several orientation-based conventional methods. Future work will be focused on reducing the feature extraction time.

Table. 2. Computational costs of various orientation-based methods in feature extraction and matching.

\begin{tabular}{|c|c|c|}
\hline Methods & Feature extraction(s) & Matching(s) \\
\hline Competitive code & 18.837 & 0.080 \\
\hline Palmcode & 1.760 & 0.204 \\
\hline Fusion code & 1.607 & 0.254 \\
\hline Ordinal code & 9.716 & 0.991 \\
\hline RLOC & 65.121 & 3.712 \\
\hline BOCV & 18.896 & 0.963 \\
\hline Half-orientation & 27.194 & 0.150 \\
\hline Proposed & 26.228 & 0.123 \\
\hline
\end{tabular}




\section{Conclusions and Prospective Research}

SPFs were used for palm-print identification as a means of producing an original recognition system in this study. SPFs were acquired using certain statistical methods. In the case of the standard PolyU database, a 0.0147 EER was obtained with the devised system in the experimental outcomes. In the current research, the proposed techniques were found to be superior. The devised system comprised a number of innovative features. Firstly, the high-level character depiction of the palm-print imaging was introduced through the application of SPFs. Secondly, to the best of the researchers' knowledge, SPFs have not been applied to palm-print characteristic imaging prior to this study, while significant identification effectiveness was realized through the use of this original system for palm-print identification. Two prospective research paths may now be followed: one path involves the identification of additional SPFs for palm-print identification, while the other involves the investigation of image improvement methods for improving the palm print representation quality.

\section{References}

Achanta R., A. Shaji, K. Smith, A. Luchi, P. Fua, and S. Susstrunk. 2012. Slic superpixels compared to state-of-the-art superpixel methods. IEEE Transactions on Patter Analysis and Machine Intelligence. 34(11):2274-2282.

Aksoy S., and R. Haralick. 2000. Feature normalization and likelihood-based similarity measures for image retrieval. Pattern Recognition Letters. Special Issue on Image and Video Retrieval.

Fei L., Y. Xu, and D. Zhang. 2016. Half-orientation extraction of palmprint features. Pattern Recognition Letters. 69:35-41.

Guo, Z., D. Zhang, L. Zhang, and W. Zuo. 2009. Palmprint verification using binary orientation cooccurrence vector. Pattern Recognition Letters. 30(13):1219-1227.

Han C. C., H. L. Chengb, C. L. Linb, and K. C. Fanb. 2003. Personal authentication using palm-print features. Pattern Recognition. 36(2):371-381.

Hanmandlu M., A. Gureja, and A. Jain. 2010. Palm print recognition using Local Binary Pattern operator and support vector machines. In Proceedings of the 2010 International Conference on Signal and Image Processing, 158-162. Chennai.

Huang D. S., W. Jia, and D. Zhang. 2008. Palmprint verification based on principal lines. Pattern Recognition. 41(4):1316-1328.

Huang G. B., Q. Y. Zhu, and C. K. Siew. 2006. Extreme learning machine: theory and applications. Neurocomputing. 70(1):489-501.

Jia W., D. S. Huang, and D. Zhang. 2008. Palmprint verification based on robust line orientation code. Pattern Recognition. 41(5):1504-1513.

Jing X. Y., and D. Zhang. 2004. A face and palmprint recognition approach based on discriminant DCT feature extraction. IEEE Transactions on Systems, Man, and Cybernetics. 34:2405-2415. 
Kale K. V., P. D. Deshmukh, S. V. Chavan, M. M. Kazi, and Y. S. Rode. 2013. Zernike moment feature extraction for handwritten Devanagari compound character recognition. In Proceedings of the 2013 Science and Information Conference, 459-466. London.

Kong A., D. Zhang, and M. Kamel. 2006. Palmprint identification using feature-level fusion. Pattern Recognition. 39(3):478-487.

Kong, A. W. K., and D. Zhang. 2004. Competitive coding scheme for palmprint verification. In: Pattern Recognition, 2004. ICPR 2004. In Proceedings of the 17th International Conference on. IEEE, 520-523.

Kumar A., and H. C. Shen. 2004. Palmprint identification using PalmCodes. In: Image and Graphics (ICIG'04), Third International Conference on. IEEE, 258-261.

Laadjel M., A. Bouridane, F. Kurugollu, and S. Boussakta. 2008. Palmprint recognition using fishergabor feature extraction. In Proceedings of International Conference on Acoustics, Speech, and Signal Processing, 1709-1712.

Lu G., D. Zhang, and K. Wang. 2003. Palmprint recognition using eigenpalms features. Pattern Recognition Letters. 24(9-10): 1463-1467.

Nanni L., and A. Lumini. 2008. Wavelet decomposition tree selection for palm and face authentication. Pattern Recognition Letters. 29(3):343-353.

Pan X., and Q. Q. Ruan. 2008. Palmprint recognition using gabor feature-based (2D) $2 P C A$. Neurocomputing. 71(13-15):3032-3036.

Ribaric S., and I. Fratric. 2005. A biometric identification system based on eigenpalm and eigenfinger features. IEEE Transactions on Pattern Analysis and Machine Intelligence. 24:1698-1709.

Shang L., D. S. Huang, J. X. Du, and C. H. Zheng. 2006. Palmprint recognition using fast ICA algorithm and radial basis probabilistic neural network. Neurocomputing. 69(13-15):1782-1786.

Sun Z., T. Tan, Y. Wang, et al. 2005. Ordinal palmprint representation for personal identification [represention read representation]. In: Computer Vision and Pattern Recognition, 2005. CVPR 2005. IEEE Computer Society Conference on. IEEE, 279-284.

Trémeau A., and P. Colantoni. 2000. Regions adjacency graph applied to color image segmentation. IEEE Transactions on Image Processing. 9(4):735-744.

Wu X., D. Zhang, and K. Wang. 2003. Fisherpalms based palmprint recognition. Pattern Recognition Letters. 24(15):2829-2838.

Wua X., D. Zhang, K. Wanga, and B. Huanga. 2004. Palmprint classification using principal lines. Pattern Recognition. 37(10):1987-1998.

Zhang, D., Guo, Z., Lu, G., Zhang, L., \& Zuo, W. 2010. An online system of multispectral palmprint verification. IEEE transactions on instrumentation and measurement, 59(2), 480-490.

Zhang, D., W. K. Kong, J. You ,2003. Online palmprint identification. IEEE Transactions on Pattern Analysis and Machine Intelligence. 25(9):1041-1050. 
Zhao Z. Q., D. S. Huang, and W. Jia. 2007. Palmprint recognition with 2DPCA+PCA based on modular neural networks. Neurocomputing. 71(1-3):448-454. 